\title{
Pure Motor Stroke Due to Vertebral Artery Dissection
}

\author{
Stephen J. Phillips, William J. Maloney and Jean Gray
}

\begin{abstract}
A 39-year-old man presented with a pure motor stroke 9 days after cervical chiropractic manipulation. Computerised tomographic scanning showed a pontine infarct. Cerebral angiography showed changes consistent with the diagnosis of bilateral vertebral artery dissection. It is postulated that the infarct resulted from artery-to-artery embolism.
\end{abstract}

RÉSUMÉ: Ictus moteur pur dû à la dissection des artères vertébrales Un homme âgé de 39 ans s'est présenté avec un ictus moteur pur survenu 9 jours après des manipulations cerviales chiropractiques. Le CT scan a montré un infarctus au niveau du pont. Une angiographie cérébrale a montré des changements compatibles avec un diagnostic de dissection bilatérale des artères vertébrales. Nous postulons que l'infarctus est le résultat d'une embolie d'artère-àartère.

Can.J. Neurol. Sci. 1989; 16:348-351

Fisher has described more than 20 clinical syndromes of lacunar infarction, 1 i.e. small deep cerebral infarcts due to hypertension-associated arteriolar disease. However, it has become increasingly apparent that clinical findings do not reliably indicate the site of the underlying brain lesion or its pathogenesis. Pure motor stroke 1,2 (hemiparesis without associated sensory deficit, homonymous hemianopia, aphasia, or agnosia) - one of the commonest lacunar syndromes - has been described in association with: lacunar infarcts in the internal capsule or basis pontis; ${ }^{2}$ infarction of the cerebral cortex, centrum semiovale, medullary pyramid, and cerebral peduncle;3,4,5,6 small hemorrhages involving the internal capsule, putamen, and basis pontis; ${ }^{7}$ saccular basilar artery aneurysm; 8 brain abscess; ${ }^{9}$ cysticercosis; ${ }^{10}$ and primary or metastatic brain tumour. ${ }^{11}$ A recently reported study of a new model of stroke in the rat suggests that small deep infarcts may be produced by platelet emboli from the cervical carotid artery. 12 Here we report a patient who had a pure motor stroke due to a pontine infarct caused by a presumed embolus from a vertebral artery dissection.

\section{Case Report}

A 39-year-old man experienced the sudden onset of dizziness, speech disturbance, and left-sided weakness while hurrying to work on April 15, 1988. He was transferred from his local hospital to Camp Hill Hospital on April 19.

Nine days before the onset of these symptoms he completed a 10 day "course" of chiropractic treatment for a painful, stiff neck which he had had for about 6 weeks. Otherwise, he had been fit and well. There was no past history of migraine, neurological symptoms, cardiovascular disease, hypertension, diabetes, alcohol abuse, or use of tobacco,

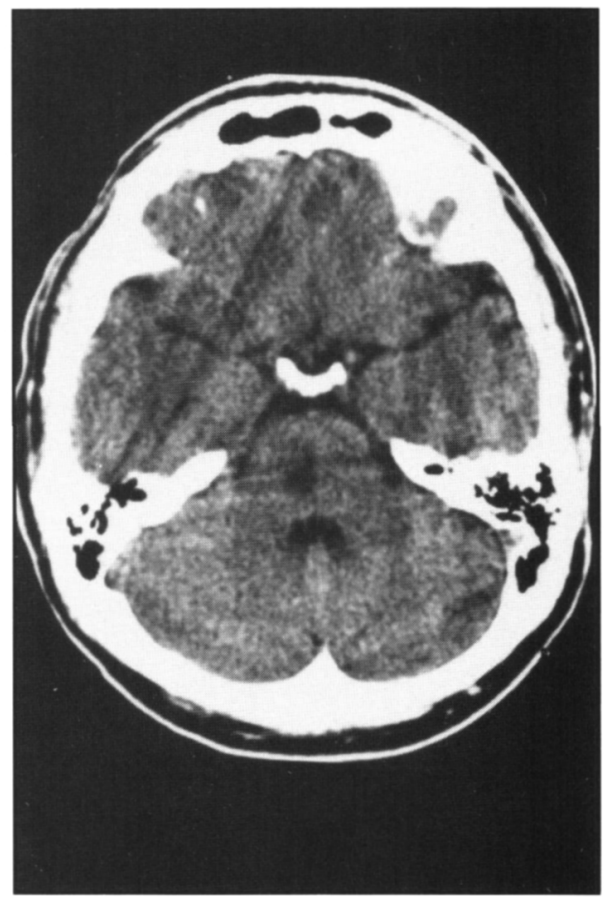

Figure I - Unenhanced CT scan showing an infarct in the right side of the pons.

From the Department of Medicine, Camp Hill Hospital (J.G., S.J.P.), and Department of Radiology, Victoria General Hospital (W.J.M.), and Dalhousie University, Halifax

Received November 17, 1988. Accepted in final form April 24, 1989

Reprint requests to: Dr. S. Phillips, Department of Medicine, Camp Hill Hospital, 1763 Robie Street, Halifax, Nova Scotia, Canada B3H 3G2 
cocaine or other sympathomimetic drugs. Family history was negative for premature vascular disease. He was not taking medications.

At the time of admission to our hospital, the general medical examination was unremarkable. Features of Marfan's syndrome were absent. While in hospital, his systolic blood pressure ranged between 110-140 $\mathrm{mm} \mathrm{Hg}$, and the diastolic between $70-80 \mathrm{~mm} \mathrm{Hg}$. No cardiac abnormalities were detected, and there were no bruits over the head and neck. Neurological examination showed a left hemiparesis involving face, arm, and leg. No other cranial nerve signs were detected. Speech and language were normal. There were no cerebellar or sensory signs. His neurological deficit was moderately severe; he could not walk, and had no useful function in the left upper extremity, i.e. grade 6 on the Stroke Severity Scale. ${ }^{13}$

The following investigations were normal or negative: hemoglobin, hematocrit, platelet count, ESR, serum proteins, serum protein electrophoresis, VDRL, chest X-ray, and ECG. Plain films of the cervical spine showed no evidence of congenital anomaly, subluxation, arthritic or degenerative changes. An echocardiogram showed prolapse of the anterior leaflet of the mitral valve.

A computerised tomographic (CT) scan done 6 days after stroke onset was normal. A CT scan done 18 days after stroke onset showed a right-sided mid-pontine infarct (Figure 1).

Four-vessel cerebral angiography was performed 13 days after stroke onset. The extracranial carotid arteries and the intracranial circulation were normal. Both posterior cerebral arteries filled from the carotid system. The left vertebral artery tapered to an occlusion in the foramen transversarium at the level of $\mathrm{C} 6$ (Figure 2). There was collateral flow to the distal left vertebral artery via branches of the left occipital artery. The right vertebral artery was irregularly stenosed throughout much of its course in the foramen transversarium (Figure 3 ). The distal right vertebral artery and the basilar artery appeared normal. The angiographic appearances were considered indicative of bilateral vertebral artery dissection.

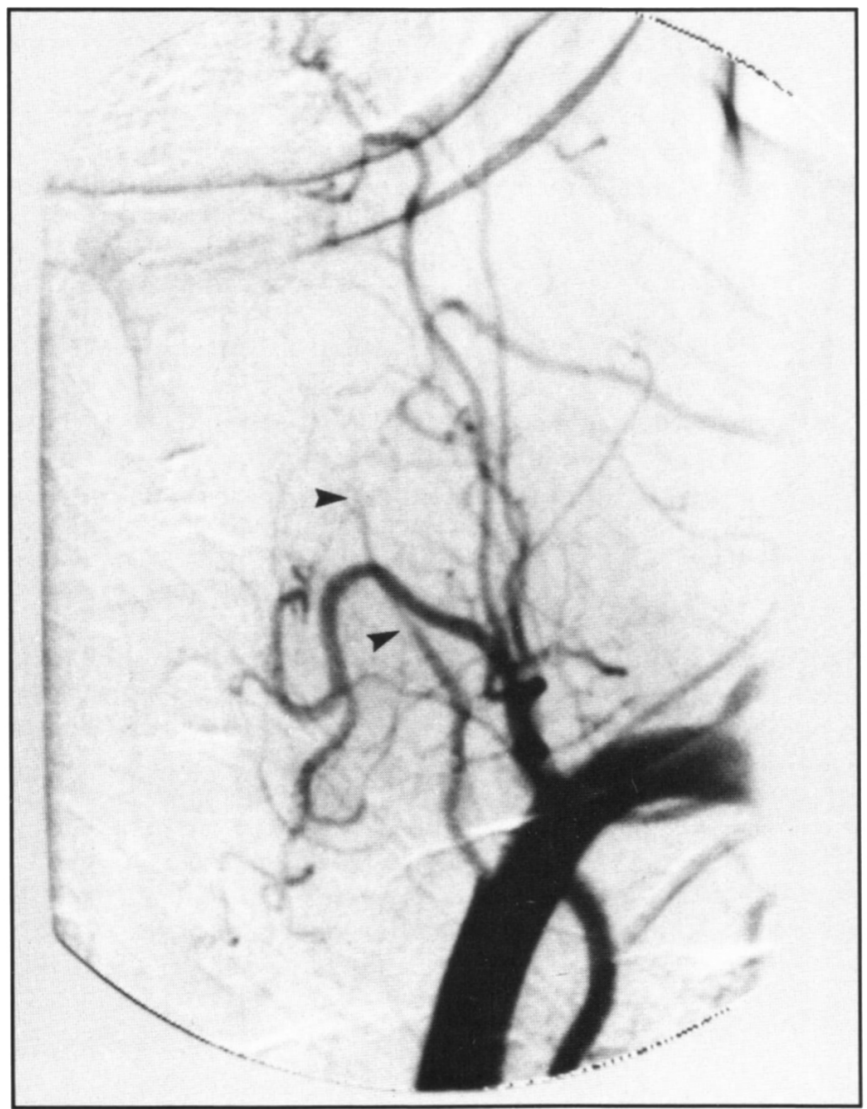

Figure 2 - Cerebral angiogram. Left subclavian injection showing a tapering stenosis (lower arrowhead), and occlusion (upper arrowhead) of the proximal left vertebral artery.

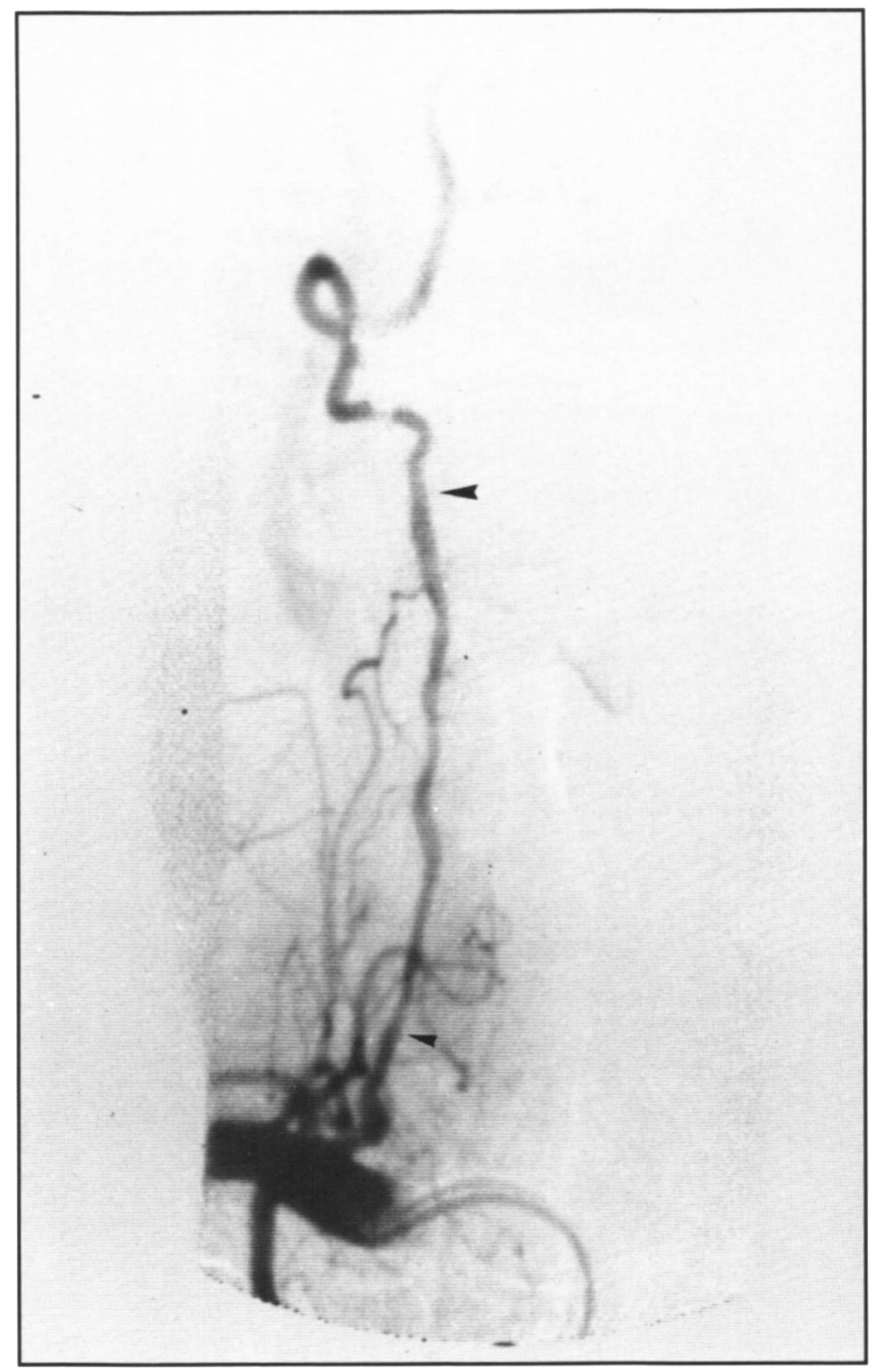

Figure 3 -Cerebral angiogram. Right subclavian injection showing a long ribbon-like narrowing of the right vertebral artery (benteen arrowheads).

During a 16-day stay in hospital he received daily occupational therapy and physiotherapy. At the time of discharge he still had a grade 6 neurological deficit on the Stroke Severity Scale; ${ }^{13}$ but his level of functioning had improved. He was able to walk with the assistance of a drop-foot splint and a cane, and opposition movements had begun to return in the left hand.

\section{Discussion}

Head and neck trauma is an important cause of cerebral infarction in young adults. ${ }^{14.15}$ Cervical chiropractic manipulation has been linked with dissections of the cervico-cerebral arteries, particularly the extracranial vertebral artery. 16-19 Vertebral artery dissections are typically bilateral in symptomatic cases. They can occur at any point in the course of the vertebral artery, but are most frequent at the $\mathrm{C} 1-\mathrm{C} 2$ and $\mathrm{C} 6$ levels, presumably because the artery is most subject to mechanical stress at these points. 19 Nucho-occipital pain and the lateral medullary syndrome are the commonest clinical manifestations. $18,20,21$ The "locked-in" syndrome, 18,22 and fatal brainstem infarction 17 have also been reported in association 
with vertebral artery dissections. Subarachnoid hemorrhage can result from dissection of the intracranial vertebral artery because this type of dissection tends to occur in the subadventitial plane. 23.24

We believe that our patient had bilateral vertebral artery dissections induced by cervical chiropractic manipulation. Atypical features were the absence of neck pain at the onset of neurological symptoms, and the 9-day delay between the last chiropractic treatment and the stroke. However, these features do not cast serious doubt on the diagnosis because painless dissections are known to occur, and the interval between trauma and stroke may vary from hours to several days. ${ }^{16}$ Angiographic diagnosis of vertebral artery dissection is presumptive, unless a pseudoaneurysm or double-lumen is demonstrated. ${ }^{19}$ These abnormalties are most likely to be demonstrated by early angiography; our patient was studied 13 days after stroke-onset. The long ribbon-like narrowing of the right vertebral artery, and the tapering stenosis and occlusion of the left vertebral artery were considered diagnostic of dissection in this patient because of his age, the history of neck trauma, and the absence of any other cause for the angiographic findings. Echocardiography demonstrated the presence of mitral valve prolapse but an embolus from this source would not produce the persistent, marked, bilateral arteriopathic changes documented here.

To our knowledge, this is the first time a pure motor stroke has been associated with vertebral artery dissection. We think that the pontine infarct was probably caused by artery-to-artery embolism. This is a recognized mechanism of brainstem infarction, ${ }^{25,26}$ and our investigations failed to provide a viable alternative explanation.

In his description of the "top of the basilar" syndrome, Caplan ${ }^{27}$ commented that since the basilar artery is widest at its origin and tapers distally, an embolus small enough to traverse the vertebral artery would ordinarily not block the basilar artery except distally. However, the present case and recent experimental evidence ${ }^{12}$ suggest that small-diameter penetrating cerebral arteries can be embolized by platelet thrombi from lesions situated in more proximal segments of the arterial tree. We admit that the evidence on which our conclusions are based is circumstantial, but direct proof of such a stroke-mechanism would be difficult or impossible to obtain, even at autopsy.

Recognition of this mechanism of stroke is important both from the clinical and scientific points of view. 28 Infarcts in the territory of end-arteries are not always due to small-vessel disease. ${ }^{29.30}$ The absence of pre-existing hypertension should prompt investigation for an alternative explanation, as it does in the normotensive patient who has a spontaneous intracerebral hemorrhage. ${ }^{31.32}$ Precise definition of the mechanism of stroke is necessary for rational therapy, ${ }^{33}$ and is especially important in young patients and individuals who have mild strokes. In research studies it is desirable to categorize strokes according to mechanism of causation; patients who have small deep cerebral infarcts do not form a pathogenetically homogeneous group.

The management of dissections is controversial. 16 Antiplatelet agents or anticoagulants are often used, provided that intracranial vertebral artery dissection and subarachnoid hemorrhage have been excluded, but it is not known whether these drugs influence outcome. We did not treat our patient with these drugs because 4 days had elapsed between stroke onset and admission to Camp Hill Hospital, his neurological deficit had already started to improve, the vertebral artery injury was caused by an avoidable form of trauma, and there was no evidence of an underlying arteriopathy that could predispose to recurrent dissection.

\section{ACKNOWLEDGEMENT}

Dr. Phillips receives support from the W. Garfield Weston Foundation of Toronto, and the Nova Scotia Heart Foundation.

\section{REFERENCES}

1. Fisher CM. Lacunar stokes and infarcts: A review. Neurology 1982; 32: 871-876.

2. Fisher CM, Curry HB. Pure motor hemiplegia of vascular origin. Arch Neurol 1965; 13: 30-44.

3. Chokroverty S, Rubino FA, Haller C. Pure motor hemiplegia due to cerebral infarction. Arch Neurol 1977; 34: 93-95.

4. Rascol A, Clanet M, Manelfe C, et al. Pure motor hemiplegia: CT study of 30 cases. Stroke 1982; 13: 11-17.

5. Ropper AH, Fisher CM, Kleinman GM. Pyramidal infarction in the medulla. A case of pure motor hemiplegia sparing the face. Neurology 1979; 29: 91-95.

6. Ho KL. Pure motor hemiplegia due to infarction of the cerebral peduncle. Arch Neurol 1982; 39: 524-526.

7. Mori E, Tabuchi M, Yamadori A. Lacunar syndrome due to intracerebral hemorrhage. Stroke 1985; 16: 454-459.

8. Fisher M, Smith TW, Jacobs R. Pure motor hemiplegia secondary to a saccular basilar artery aneurysm. Stroke 1988; 19: 104-107.

9. Weintraub MI, Glaser GH. Nocardial brain abscess and pure motor hemiplegia. NY State J Med 1970; 70: 2717-2721.

10. Barinagarrementeria $\mathrm{F}$, Del Brutto $\mathrm{OH}$. Neurocysticercosis and pure motor hemiparesis. Stroke 1988; 19: 1156-1158.

11. Fisher M, Recht LD. Brain tumor presenting as an acute pure motor hemiparesis. Stroke 1989; 20: 288-291.

12. Futrell N, Watson BD, Dietrich D, et al. A new model of embolic stroke produced by photochemical injury to the carotid artery in the rat. Ann Neurol 1988; 23: 251-257.

13. The EC/IC Bypass Study Group. The international cooperative study of extracranial/intracranial arterial anastomosis (EC/IC Bypass Study): methodology and entry characteristics. Stroke 1985; 16: 397-406

14. Hilton-Jones D, Warlow CP. The causes of stroke in the young. J Neurol 1985; 232: 137-143.

15. Bogousslavsky J, Regli F. Ischemic stroke in adults younger than 30 years of age: cause and prognosis. Arch Neurol 1987; 44: $479-482$.

16. Hart RG, Easton JD. Dissections and trauma of cervico-cerebral arteries. In: Barnett HJM, Mohr JP, Stein BM, Yatsu FM, eds. Stroke: Pathophysiology, Diagnosis, and Management. Churchill Livingstone, 1986: 775-788.

17. Dunne JW, Conacher GN, Khangure $M$, et al. Dissecting aneurysms of the vertebral arteries following cervical manipulation: a case report. J Neurol Neurosurg Psychiatry 1987; 50: 349-353.

18. Mas J-L, Bousser M-G, Hasboun D, et al. Extracranial vertebral artery dissections: a review of 13 cases. Stroke 1987; 18: 10371047.

19. Hart RG. Vertebral artery dissection (editorial). Neurology 1988; 38: 987-989.

20. Caplan LR, Zarins CK, Hemmati M. Spontaneous dissection of the extracranial vertebral arteries. Stroke 1985; 16: 1030-1038.

21. Mokri B, Houser OW, Sandok BA, et al. Spontaneous dissections of the vertebral arteries. Neurology 1988; 38: 880-885. 
22. Horn SW. The "locked-in" syndrome following chiropractic manipulation of the cervical spine. Ann Emerg Med 1983; 12: 648-650.

23. Deck JHN. Pathology of spontaneous dissection of intracranial arteries. Can J Neurol Sci 1987; 14: 88-91.

24. Caplan LR, Baquis GD, Pessin MS, et al. Dissection of the intracranial vertebral artery. Neurology 1988; 38: 868-877.

25. Fisher CM, Karnes WE. Local embolism. J Neuropathol Exp Neurol 1965; 24: 174-175.

26. Pessin MS, Daneault N, Kwan ES, et al. Local embolism from vertebral artery occlusion. Stroke 1988; 19: 112-115.

27. Caplan LR. "Top of the basilar" syndrome. Neurology 1980; 30: $72-79$.
28. Bamford JM, Warlow CP. Evolution and testing of the lacunar hypothesis. Stroke 1988; 19: 1074-1082.

29. Fisher CM, Caplan LR. Basilar artery branch occlusion: a cause of pontine infarction. Neurology 1971; 21: 900-905.

30. Fisher CM. Bilateral occlusion of basilar artery branches. J Neurol Neurosurg Psychiatry 1977; 40: 1182-1189.

31. Kase CS. Intracerebral hemorrhage: non-hypertensive causes. Stroke 1986; 17: 590-595.

32. Caplan L. Intracerebral hemorrhage revisited. Neurology 1988; 38: 624-627.

33. Caplan LR. TIAs: we need to return to the question, "What is wrong with Mr. Jones?" Neurology 1988; 38: 791-793. 